were 1) to determine the prevalence of PLEs in a clinical sample of non-psychotic young people; 2) to investigate the stability of these PLEs over a 6-month period and 3 ) to examine the associations between PLEs, psychiatric morbidity and psychosocial functioning.

Methods: Consecutive referrals to a youth psychiatric service $(n=140)$ were assessed to measure PLEs, psychiatric morbidity and psychosocial functioning. These assessments were undertaken at the time of referral, 3 and 6 months. The PLE data were factor analyzed to determine whether there were different subtypes of these experiences. The associations between PLEs, psychiatric morbidity and psychosocial functioning were also examined.

Results: Almost all of the sample (98.6\%) reported experiencing at least one of the PLEs at least 'sometimes' in their lifetime. PLEs were also highly prevalent at 3 and 6 months. Three subtypes of PLEs were identified: bizarre experiences, persecutory ideas and magical thinking. Bizarre experiences and persecutory ideas were associated with poor functioning.

Conclusions: Bizarre experiences and persecutory ideas may be more malignant forms of psychotic symptoms as they are associated with current disability and may confer increased risk of development of full-blown psychotic disorder.

\section{Investigating the 'trill of suspirality': thought disorder as an emergent property of conversational interaction}

\section{R Barrett', J Crichton²}

'University of Adelaide, Discipline of Psychiatry; and 'University of South Australia, Adelaide, Australia

Background: Formal thought disorder is an important although not pathognomonic clinical feature of schizophrenia. The clinical and neurocognitive literature on thought disorder, although vast, is limited to the extent that it draws on a model of thought that can be traced to the English Enlightenment and Locke's theory of ideas and their associations, itself based on his clinical experience and the influence of Newton's physics of atoms and forces. We propose an alternative model that draws on recent work in linguistics. By giving greater emphasis to the interactional aspects of language, it enables a sharper focus on thought disorder as an emergent property of conversation.

Methods: Conversational interaction between patients with identified thought disorder and psychiatrist is analyzed. The analysis focuses on agency as it manifests in the management of turn taking (drawing on conversational analytic techniques) and in transitivity structures within clauses (drawing on systemic functional grammar).
Results: Patients are accomplished agents in the management of their part of turn taking. However, in representing themselves as agents in language, they transfer their agency onto other phenomena in their lived world, including inanimate objects and objects that are coined through neologisms.

Conclusions: This novel method of investigating thought disorder as fraught conversational interaction provides for the possibility of sharpening the definition of thought disorder as a construct, with attendant benefits both for clinical detection and for neurobiological research.

\section{Association of cytokines and cognitive functioning in the elderly general population}

\section{B Baune', K Berger ${ }^{2}$}

'School of Medicine, James Cook University, Townsville, Australia; and 2Institute of Epidemiology and Social Medicine, University of Muenster, Muenster, Germany

Background: The relationship between cytokines as markers of systemic inflammation and cognitive decline in the elderly has been established, whereas little knowledge has been gained as to whether domains of cognitive functioning are affected by cytokines. This study is to investigate the associations between circulating cytokines and specific neuropsychological domains of cognitive functioning (memory, processing speed and motor function) and general cognitive function (MMSE) in healthy elderly individuals.

Methods: In a cross-sectional study of 369 community-dwelling elderly subjects, we examined the relationship between serum interleukin (IL)-1beta, sIL-4R, IL-6, IL-8, IL-10, IL-12 and tumor necrosis factor-alpha concentrations and cognitive performance using an extensive cognitive test battery.

Results: Multivariate analysis showed that increased serum concentrations of IL-8 were associated with poor performance in the memory and speed domain and in motor function. Serum concentrations of IL-1beta, IL6 and IL-10 were associated with impairments in specific domains of cognitive functioning, but these effects disappeared after Bonferroni's correction for multiple testing. Global cognitive functioning, as measured with MMSE, was not affected by cytokine levels.

Conclusions: This study suggests an association between circulating IL-8 concentrations and cognitive dysfunction in the elderly. An interaction between this cytokine and glial cells may help explain the pathophysiological mechanisms leading to cognitive impairment in our study group. 